\title{
Study on Comprehensive Evaluation Model of E-commerce Platform based on Fuzzy Theory
}

\author{
Tingting Zhang ${ }^{1, \text { a }}$ \\ ${ }^{1}$ Beijing Polytechnic College, Beijing, China, 10042 \\ a email
}

Keywords: E-commerce, Fuzzy Theory, Comprehensive Evaluation Model

\begin{abstract}
The advantage of e-commerce is that it is connected directly to the producers and consumers goods to consumers directly from the manufacturers. It not only greatly reduce the cost of middlemen in the traditional sales model, and allow users to stay at home can be a variety of consumer shopping. Clearly, consumers always want to find the best e-commerce platform, in order to obtain the best service and the best value. E-commerce platform operators also need to know the extent of the site popular with users, and the success and shortcomings of the site, in order to further improve. To solve the above problems, this paper aims to establish a scientific evaluation system to explore the comprehensive evaluation of the application of e-commerce platform level.
\end{abstract}

\section{Introduction}

E-commerce in the early 1990s in Europe and has the rise of a new mode for commercial transactions. Its characteristics are in the Intemet environment to achieve consumer online shopping, online transactions and online electronic payments between merchants. Traditional e-commerce business processes electronic, digital, electronic flow instead of real logistics, greatly reducing the human and material consumption, reduce transaction costs; the other hand, it breaks through the limitations of time and space, significantly improve the efficiency of the transaction. E-commerce has the openness and global characteristics for enterprises and individuals to create more trade opportunities. It has the unique circulation patterns, reducing intermediate links, and changed the way the social economy to some extent.

In traditional business, the two sides traded through face to face contact, recommendation, suggesting an initial trust, etc., and the use of mechanisms to protect the laws, regulations and other transactions, and maintain the trust relationship between them. With the development of information technology, the rise of e-commerce has not only changed the traditional business model of business activities, and accelerate the transformation of the content and form of trust. In a network environment, the degree of separation of time and space sides of the transaction further intensified, both sides can not be identified by way of face to face contact with a false identity, the initial formation of trust. Currently, e-commerce and related facilities, and other laws and regulations is not perfect, so that the original contract law and other laws and regulations can not be implemented effectively monitor online transactions, credit information disclosure mechanism is not carried out under laws and regulations, it is difficult to trust the network the long-term stable development of reliable legal protection, leading to a variety of illegal activities false trading, counterfeiting, phishing, and other online auctions to drive up the standard occur. Lack of trust in e-commerce e-commerce has become an important reason hinder rapid and healthy development.

\section{Fuzzy Theory Foundation}

Human activities include both understand the world and transform the world, one based on observations of the objective world, knowledge, and make the appropriate decisions and actions, this process can be abstracted as a mapping from the input space to output space, complete this many ways or methods, such as linear systems, expert systems, difference equations, check the value of multi-dimensional tables, neural networks and fuzzy systems map. Wherein the fuzzy system is one of the fastest and most convenient way, because the fuzzy system description and 
characterization of the system is based on natural language, and natural language of mankind for thousands of years after the formation of the historical development of mankind is undoubtedly most convenient and effective means of expression.

Fuzzy Systems has been able to quickly and easily deal with the problem description and based on the following facts: Fuzzy logic is based on natural language description; fuzzy logic can be based on the experience of experts; fuzzy logic allows the use of inaccurate data; - fuzzy logic conceptually easy to understand; can complex nonlinear function of a fuzzy logic modeling arbitrary.

Fuzzy system is based on natural language, and natural language often used in some vague concepts such as inflation, the market slump, high prices and good quality. How to describe these fuzzy concepts and analyze them reasoning which is the fuzzy sets and fuzzy logic to solve the problem.

Fuzzy set is a set of boundary is not clear, Fuzzy Sets and ordinary collection of different but linked. For ordinary collection, any element or part of the collection, the collection does not belong to either all or nothing, with a clear and precise boundaries; and for fuzzy sets, an element can be both part of the collection does not belong to the collection, Both A and B, the boundary is not clear or blurred boundaries.

Since the classic rally can only have a clear concept of the extension, not the performance of fuzzy concept, and therefore on the classic rally in front of the fuzzy concept powerless. Thus, the quantitative performance of fuzzy concept and research has the objective laws of fuzziness of the object, it is necessary to promote the concept of the classic collection. Built on fuzzy sets based on fuzzy logic, the truth of any statement or proposition is a certain degree of authenticity, compared with the established in the general collection based on Boolean logic, fuzzy logic is a generalization of logic. In Boolean logic, any statement or proposition only two values, namely logical true and false logic, common 1 represents a logically true 0 means logically false. In fuzzy logic, statements or propositions true and false values except 0 and 1 , but may take any value between 0 and 1 , such as 0.75 , that is, the extent to which a statement or proposition is true or false, for example. The concept of the elderly in general need to define a clear set of boundaries, such as the elderly aged over 60, while in fuzzy sets, defined set of elderly without a clear boundary, is over 60 years old, 58-year-old man also belongs 40 -year-old to a certain extent, also belong to the elderly, but they belong to different extent of this collection of old has been. In order to quantitatively describe fuzzy concepts and fuzzy objects, the US computer and cybernetics expert Professor I.A.Zadeh put forward the important concept of fuzzy sets in 1965. The basic idea is: to be expanded affiliation classic collection, so that the elements right. set. By the degree of membership can only take two values 0 and 1 can be generalized to take the unit interval $[0,1]$ any of a number, in order to achieve quantitatively characterize the ambiguity object.

\section{Overall Evaluation based on Ahp and Fuzzy Evaluation Model of E-commerce Platform}

To quantitatively determine the weight, in our view, the analysis can be hierarchical model. Analytic Hierarchy Process is home TLsaaty American operations research, who in the early seventies and has been presented widely, AHP is one of the mathematical tools of systems analysis, qualitative analysis and quantitative analysis will combine the people hierarchical thinking process, principled, quantitative, on the merits of the decision-making program to sort, practical, systematic and simplicity and other characteristics.

Characteristics of China's e-commerce platform metrics to determine the index weight is the main reason for this paper, using analytic hierarchy process. Index system involving a number of free and interact, but some indicators can be broken down into different sub-index, which constitute a complex system of the tree, this tree system not only provides the structural basis for the analytic hierarchy process, but also increases the evaluation system in the practical application flexibility.

Establish a hierarchical model based on the study and research goals. After-depth analysis facing the factor in question contained divided into different levels, and explain affiliation level hierarchical structure and factors used in block diagram form, in general, may want to research the 
problem into three basic levels, the first, the highest level; represents the overall goal of the purpose of solving the problem, namely the level of analysis to be achieved. Second, the intermediate layer; represents various measures, programs to achieve the overall goal of the predetermined involved in the middle part of the intermediate layer can be divided into general policy level, constraints, rule, etc., depending on the problem to be solved may be. Third, the lowest level; pledged to solve the problem of the choice of a variety of measures, programs and the like.

First, when the difference between the real thing to be compared in terms of the properties we are considering having the same order of magnitude or very close to, the qualitative makes sense, but also have a certain degree of clarity. Second, five levels can be a good thing that distinguishes certain aspects of the property, when you need more precision, you can also make comparisons between adjacent levels, so that a total of nine values, they have coherence, in practice, it can be a good application. Third, the social survey shows that in general, most people need seven point scale to differentiate differences in quality or degree of importance between things. Meanwhile, the survey also shows that psychology, 7 persons 2 project psychology limit. If we take 7 persons 2 Inso-by comparison, the differences between them can be up to 9 digits shown.

\section{Fuzzy Comprehensive Evaluation}

As an application of fuzzy comprehensive evaluation method of fuzzy mathematics, was first used by Chinese scholars Wang Peizhuang proposed [28]. In real life, the same thing or phenomena tend to have a variety of properties, so the time to evaluate things, we must take into account all aspects, have to make a comprehensive consideration of a number of factors. Fuzzy comprehensive evaluation is an effective tool to solve such problems. Results indicator system has multilevel nature, received preliminary results of the evaluation in accordance with the basic index layer, and then obtain the basic results of the evaluation based on the corrected correction indicators, on the basis of basic evaluation on the combination of the basic conclusions Judgments further supplement to give comprehensive evaluation. E-commerce platform evaluation is a complex system problem, only from a different perspective to assess, in order to achieve a comprehensive and objective evaluation. In this case, this paper introduces fuzzy comprehensive evaluation method.

Fuzzy comprehensive evaluation result itself is not a single point value, but a vector, and this vector is a fuzzy subset can accurately portray the thing itself fuzzy situation. And fuzzy comprehensive evaluation results can be further processed to give a series of comprehensive reference information, such as the assessment by the corresponding level object, fuzzy vector corresponding to a single value, and so on.

When the indicators themselves from the perspective of the importance of determining the weights, usually right across Evaluation System weight as a whole. But if things are more complex evaluation, property relatively long time, the index will contain more bound to the weight of each index weight is small. Differences between the importance of the indicators is not easy to be reflected. If all indicators hierarchical processing, each of the inner-level indicators will make less, but the importance of the difference is relatively easy to determine. Thus, the more levels and more complex things, the application of multi-level fuzzy comprehensive evaluation, the effect is more ideal.

Weighting Fuzzy comprehensive evaluation of adaptability, according to different evaluators focus, the right to change evaluation indexes. It also can simultaneously use several sets of different weights of the same assessment is a comprehensive evaluation of the object to be compared. But note that the different objects to be evaluated right index must use a uniform weight, before comparing the results, if you use different weights, the results are not comparable.

\section{Conclusion}

Evaluation of e-commerce platform is a complex project, the paper discusses the survey analysis, expert evaluation and comprehensive evaluation. E-commerce platform on the index, which is the key issue in the actual work. People from a different point of view for different index system, this 
paper presents the actual situation of our evaluation system more appropriate to the level of development of China's e-commerce platform, so that more specific detailed specification of the evaluation system of e-commerce platform, hoping to It provides a useful reference for the construction and evaluation of e-commerce platform.

\section{References}

[1] Mcknight D H, Chervany N L , What Trust Means in E-Commerce Customer Relationships: An Interdisciplinary Conceptual Typology [J], International Journal of Electronic Commerce, 2002,6 (2) : 35 59 .

[2] Pavlou P A, Chellappa R K,The Role of Perceived Privacy and Perceived Security in the Development of Trust in Electronic Commerce Transactions [J], Special Issue of ISR on “Electronic Commerce Metrics,2001.

[3] Pavlou P A, Institution-Based Trust in Interorganizational Exchange Relationships: The Rolebof Online B2b Marketplaces On Trust Formation [J], Journal of strategic Information Systems,2002,11: 215 243.

[4] Tan Y -,Thoen W,An Outline of a Trust Model for Electronic Commerce [J], Applied Artificial Intelligence,2000,14: 849 862.

[5] Klang M, Who Do You Trust? Beyond Encryption, Secure E-Business [J], Decision Support System, 2001, 31.

[6] Matteo D B,Trust Generation through Extralegal Mechanisms: Reputation as a Valued Asset in Emarkets [C],16th Bled e-commerce Conference Transformation, 2003. 